\title{
Cascaded Wavelength Conversion by Four-Wave Mixing in a Strained Semiconductor Optical Amplifier at $10 \mathrm{~Gb} / \mathrm{s}$
}

\author{
Robert B. Lee, David F. Geraghty, Marc Verdiell, Member, IEEE, Mehrdad Ziari, Member, IEEE, \\ Atul Mathur, Member, IEEE, and Kerry J. Vahala, Member, IEEE
}

\begin{abstract}
We demonstrate for the first time cascaded wavelength conversion by four-wave mixing in a semiconductor optical amplifier. Bit-error-rate performance of $<10^{-9}$ at $10 \mathrm{~Gb} / \mathrm{s}$ is achieved for two conversions of up to $9 \mathrm{~nm}$ down and up in wavelength. For two wavelength conversions of $5 \mathrm{~nm}$ down and up, a power penalty of $1.3 \mathrm{~dB}$ is measured. A system of two wavelength converters spanning $40 \mathrm{~km}$ of single-mode fiber is also demonstrated.
\end{abstract}

Index Terms-Communication systems, frequency conversion, optical mixing, semiconductor optical amplifier.

A N ESSENTIAL new function required in wavelength division multiplexed (WDM) all-optical networks is wavelength conversion, the spectral translation of informationladen optical carriers. It enhances wavelength routing options and greatly improves network reconfigurability, eliminating problems associated with channel contention and wavelength reuse. Four-wave mixing (FWM) utilizing ultrafast intraband nonlinearities in semiconductor optical amplifiers (SOA's) has been studied in recent years as a means to implement this function [1], [2]. Its transparency to both the bit rate and the modulation format of the input signal is a strong advantage over other competing wavelength conversion techniques. For a single SOA wavelength converter using FWM, minimal degradation in system performance has been demonstrated previously at bit rates up to $10 \mathrm{~Gb} / \mathrm{s}$ [3]. However, a signal path in an all-optical network will contain multiple crossconnects making cascadability of any wavelength conversion technique indispensable to practical implementation in a WDM network. Cascadability has been demonstrated in an interferometric wavelength converter based on SOA's [4] and in a wavelengthtunable semiconductor laser [5]. In this letter, we present the first demonstration of cascaded wavelength conversion in a semiconductor optical amplifier by FWM.

The schematic of the experimental setup is shown in Fig. 1. In Fig. 1(a), the detailed configuration of a SOA FWM

Manuscript received January 7, 1997; revised February 18, 1997. This work was supported by DARPA under Contract DAAL 01-94-K-03430, by the National Science Foundation under Grant ECS-9412862, and by Northrop-Grumman Corporation.

R. B. Lee, D. F. Geraghty, and K. J. Vahala are with the Department of Applied Physics, California Institute of Technology, Pasadena, CA 91125 USA.

M. Verdiell, M. Ziari, and A. Mathur are with SDL, Inc., San Jose, CA 95134 USA.

Publisher Item Identifier S 1041-1135(97)04078-0. wavelength converter is shown. The pump source is a tunable external-cavity semiconductor laser. The information carrying signal is combined with the pump laser in an 80/20 bidirectional coupler and subsequently amplified in a highpower erbium-doped fiber amplifier (EDFA). Following the amplification, a wide-optical bandpass filter referred to as the amplifide spontaneous emission (ASE) prefilter, (10- and 16nm FWHM in converters 1 and 2, respectively) is used to reduce the ASE in the spectral region of the converted signal thereby providing improved optical signal-to-noise ratio (SNR) of the converted signal [6], [7]. Approximately $+13 \mathrm{dBm}$ of total optical power is input to the FWM medium, a 1-mm-long compressively strained multiple quantum-well semiconductor optical amplifier with 22-dB fiber-to-fiber small-signal gain at $300-\mathrm{mA}$ bias current. Mechanical polarization controllers are used to independently align the pump and the signal to the TE polarization of the SOA. At the output of the SOA, the converted signal is isolated by use of a $1.0-\mathrm{nm}$ optical bandpass filter (OBF).

In Fig. 1(b), the system setup for the cascaded wavelength conversion experiment is shown. In wavelength converter 1 the signal is downshifted in wavelength, and in wavelength converter 2 the signal is upshifted by an equal amount ranging from 4 to $9 \mathrm{~nm}$. The source for the signal is provided by a DFB laser at $1558.7 \mathrm{~nm}$ and modulated externally using a $\mathrm{LiNbO}_{3}$ Mach-Zehnder modulator. At the output of converter 1, the converted signal is amplified and filtered using an EDFA-OBF combination. In the experiment, a single source is divided in power to provide the pump waves for the two converters. In order to reduce the interference between the pump wave for converter 2 and the residual pump wave from converter 1 due to incomplete suppression achieved by filtering, an extra OBF is used. The $40-\mathrm{km}$ transmission link spanning the two converters is indicated by a shaded box. An EDFA-OBF pair is used to compensate for the propagation losses in the $40 \mathrm{~km}$ of dispersive single-mode fiber (SMF). The converted signal at the output of the second wavelength converter is detected using a preamplified receiver. In the receiver, the signal is amplified with a low-noise two-stage EDFA, filtered with an $\mathrm{OBF}$ and then detected using an 11-GHz bandwidth p-i-n receiver. The electrical signal is subsequently amplified by a 6-GHz electrical amplifier. A Hewlett-Packard bit-error-rate (BER) tester consisting of a pattern generator, a microwave transition analyzer and BER analyzer is used in the experiment. 


\section{Wavelength Converter}

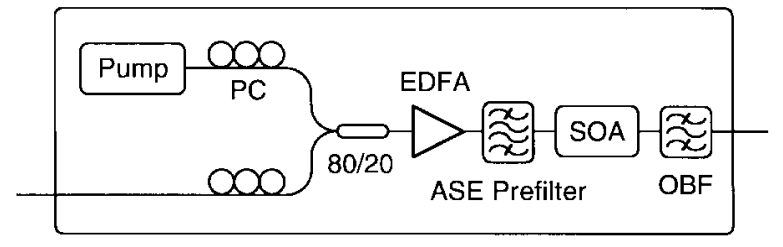

(a)

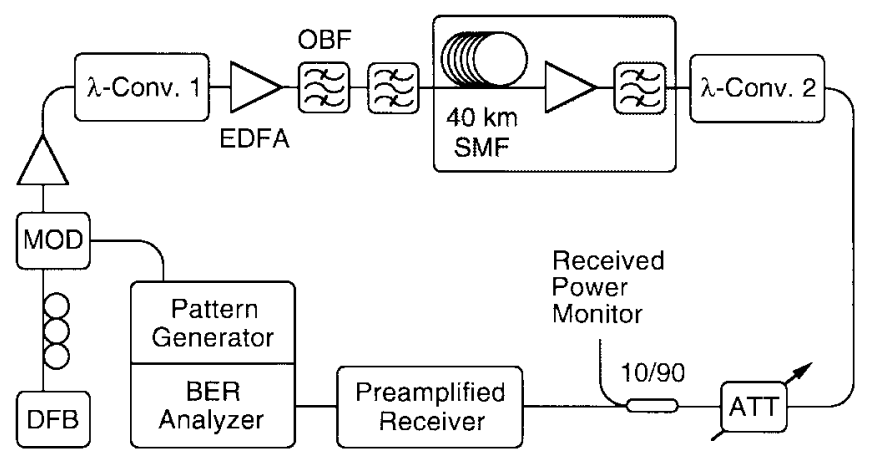

(b)

Fig. 1. (a) A schematic of the detailed configuration of a FWM wavelength converter with signal input at the left and converted signal output at the right; and (b) the experimental setup for cascaded wavelength conversion spanning $40 \mathrm{~km}$ transmission in a single-mode fiber. PC: polarization controller, EDFA: erbium-doped fiber amplifier, OBF: optical bandpass filter (1.0-nm FWHM), DFB: distributed feedback laser, MOD: $\mathrm{LiNbO}_{3}$ Mach-Zehnder modulator, ATT: variable attenuator.

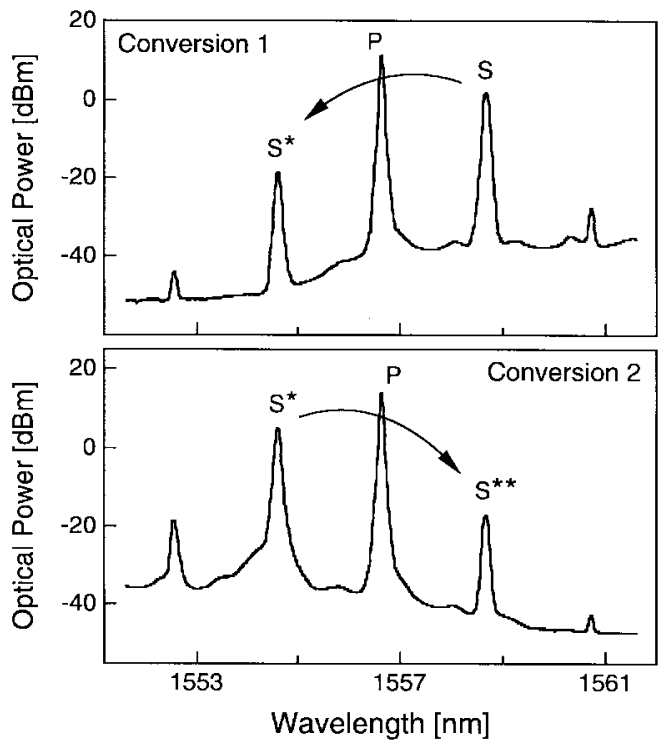

Fig. 2. Optical spectra measured using a $0.1-\mathrm{nm}$ resolution bandwidth at the output of the semiconductor optical amplifier in the indicated wavelength converter for a wavelength shift of $4 \mathrm{~nm}$.

In Fig. 2, the optical spectra measured into 0.1-nm resolution bandwidth at the output of the SOA in wavelength converters 1 and 2 for a 4-nm wavelength shift down and up, respectively, are shown. The pump is labeled $\mathrm{P}$, the original signal $S$, the converted signal $S^{*}$ and the twice-converted signal $S^{* *}$. The system performance of the converters are evaluated using a 10$\mathrm{Gb} / \mathrm{s}$ pseudorandom nonreturn-to-zero (NRZ) pattern of length $2^{31}-1$. To assess the degradation in system performance re-

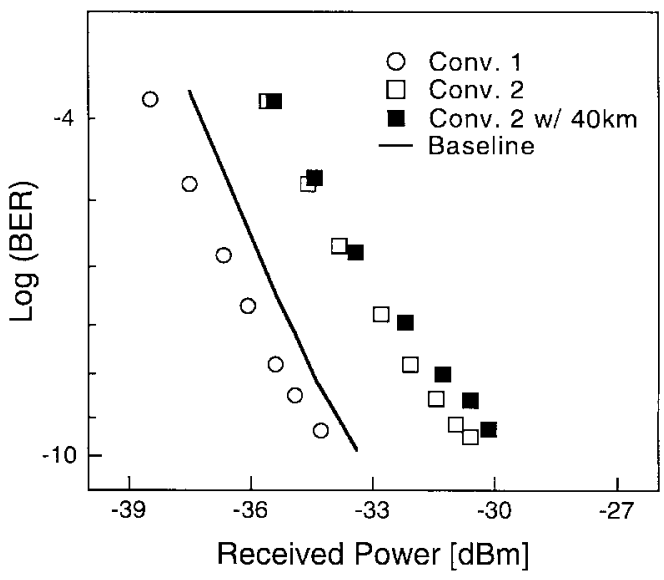

Fig. 3. BER versus received power following wavelength conversion 1 and wavelength conversion 2 with and without 40-km transmission in single-mode fiber for a wavelength shift of $4 \mathrm{~nm}$. A baseline curve for the input signal is also shown. The received power is measured on the optical spectrum analyzer using a $0.5-\mathrm{nm}$ resolution bandwidth.

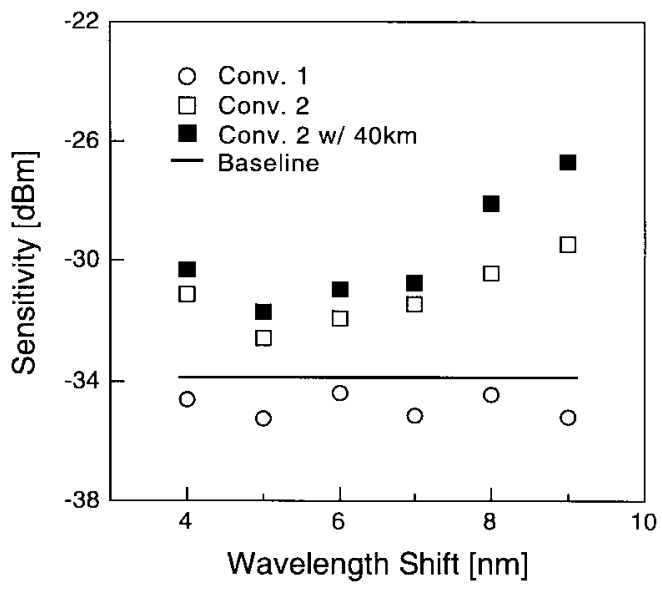

Fig. 4. Receiver sensitivity $\left(B E R=10^{-9}\right.$ ) versus wavelength shift for a single-wavelength conversion and cascaded wavelength conversion with and without $40-\mathrm{km}$ transmission. Baseline indicates the sensitivity for the original input signal.

sulting from the wavelength conversion, BER versus received power curves are measured after wavelength conversion 1 and wavelength conversion 2 with and without the 40-km transmission. In Fig. 3, the BER data are shown for the above cases corresponding to a 4-nm shift. Also shown is a baseline curve of the unconverted signal. We note that the first converted signal has a better BER performance. This results from the spectral dependence of the EDFA used in the preamplified receiver, and the dc operating point of the external modulator which is adjusted for maximum performance of the second conversion. The fixed wavelength of the DFB source does not allow for the measurement of a proper baseline for wavelength conversion 1. However, for the twice-converted signals, because they correspond exactly to the unconverted signal in wavelength, the BER data can be directly compared to the baseline shown.

The above experiment is conducted for wavelength shifts ranging from 4 to $9 \mathrm{~nm}$ at $1-\mathrm{nm}$ intervals by varying the pump wavelength. To quantify the overall system performance, 
we measure the receiver sensitivity $\left(\mathrm{BER}=10^{-9}\right)$. Fig. 4 summarizes the results of the experiment. Due to the increasing difficulty associated with suppressing the pump wave from conversion 1 for shorter wavelength shifts, the minimum in the sensitivity for conversion 2 does not occur at the shortest wavelength shift where conversion efficiency and optical SNR are maximum. For the longer wavelength shifts, the sensitivity degrades due to the decreasing optical SNR. For the case of conversion 2 with 40-km transmission, the additional decrease in the optical SNR resulting from the extra amplification stage further degrades the sensitivity. The power penalties for the optimum performance at a wavelength shift of $5 \mathrm{~nm}$ are 2.2 and $1.3 \mathrm{~dB}$ for conversion 2 with and without $40-\mathrm{km}$ transmission, respectively.

In conclusion, we have demonstrated the cascadability of wavelength conversion by FWM in an SOA. For an externally modulated signal at $10 \mathrm{~Gb} / \mathrm{s}$, a BER performance of $<10^{-9}$ and small power penalties were achieved for wavelength shifts ranging from 4 to $9 \mathrm{~nm}$. A transmission experiment was conducted using $40 \mathrm{~km}$ of SMF and small additional degradation in the BER performance was found. The results indicate the feasibility of wavelength conversion by FWM in an SOA for application in an all-optical wavelength-switched network.

\section{REFERENCES}

[1] M. C. Tatham, G. Sherlock, and L. D. Westbrook, "20 nm wavelength conversion using nondegenerate four-wave mixing," IEEE Photon. Technol. Lett., vol. 5, pp. 1303-1306, 1993.

[2] J. Zhou, N. Park, J. W. Dawson, K. J. Vahala, M. A. Newkirk, and B. I. Miller, "Efficiency of broadband four-wave mixing wavelength conversion using semiconductor traveling-wave amplifiers," IEEE Photon. Technol. Lett., vol. 6, pp. 50-52, 1994.

[3] R. Ludwig and G. Raybon, "BER measurements of frequency converted signals using four-wave mixing in a semiconductor laser amplifier at 1 , 2.5, 5, and 10 Gbit/s," Electron. Lett., vol. 30, no. 4, pp. 338-339, 1994.

[4] R. J. S. Pedersen, M. Nissov, B. Mikkelsen, H. N. Poulsen, K. E. Stubkjaer, M. Gustavsson, W. van Berlo, and M. Janson, "Transmission through a cascade of 10 all-optical interferometric wavelength converter spans at 10 Gbit/s," Electron. Lett., vol. 32, no. 11, pp. 1034-1035, 1996.

[5] H. Yasaka, H. Ishii, Y. Yoshikuni, and K. Oe, "Repeated wavelength conversion of $10 \mathrm{Gbit} / \mathrm{s}$ signal using wavelength-tunable semiconductor lasers," Electron. Lett., vol. 7, pp. 161-163, 1995.

[6] J. Zhou and K. J. Vahala, "Noise reduction in FWM wavelength converters," in Proc. CLEO'95, 1995, paper CThT1.

[7] W. Shieh and A. E. Willner, "SNR improvement of four-wave mixing wavelength shifting by noise prefiltering in a semiconductor optical amplifier," in Proc. CLEO'96, 1996, paper CThB5. 